\title{
Is it possible to identify the inguinal nerves during hernioplasty? A systematic review of the literature and meta-analysis of cadaveric and surgical studies
}

\author{
R. Cirocchi ${ }^{1}$ - B. M. Henry ${ }^{2}$ I. Mercurio ${ }^{1} \cdot$ K. A. Tomaszewski ${ }^{2} \cdot$ P. Palumbo $^{3} \cdot$ A. Stabile $^{1} \cdot$ M. Lancia ${ }^{1} \cdot$ J. Randolph $^{4}$
}

Received: 8 June 2018 / Accepted: 8 November 2018 / Published online: 20 December 2018

(c) The Author(s) 2018

\begin{abstract}
Purpose Patients who undergo inguinal hernioplasty may suffer from persistent postoperative pain due to inguinal nerve injuries. The aim of this systematic review and meta-analysis was to provide comprehensive data on the prevalence (identification rates), anatomical characteristics, and ethnic variations of the ilioinguinal (IIN), the iliohypogastric (IHN) and the genital branch of the genitofemoral (GNF) nerves.

Methods The systematic literature search was conducted using the PubMed, Scopus and Web of Science databases.

Results A total of 26 articles (5265 half-body examinations) were included in this study. The identification rate of the IIN was $94.4 \%$ (95\% CI 89.5-97.9) using a random-effects model. Unweighted multiple regression analysis showed that study sample size $(\beta=-0.74, p=.036)$ was the only statistically significant predictor of lower prevalence. The identification rates of the IHN and GNF was $86.7 \%$ (95\% CI 78.3\%-93.3\%) and 69.1\% (95\% CI 53.1\%-83.0\%) using a random-effects model, respectively. For those outcomes, a visual analysis of funnel and Doi plots indicated irregularity and provided evidence that larger studies tended to have lower identification rates. In terms of the synthesis of anatomical reference points, there was a large and statistically significant amount of heterogeneity for most outcomes.

Conclusions The identification rates of the inguinal nerves in our study were lower than reported in literature. The lowest was found for GNF, suggesting that this nerve was the most difficult to identify. Knowledge regarding the anatomy of the inguinal nerves can facilitate their proper identification and reduce the risk of iatrogenic injury and postoperative pain.
\end{abstract}

Keywords Hernioplasty $\cdot$ Iliohypogastric nerve $\cdot$ Ilioinguinal nerve $\cdot$ Genital branch of the genitofemoral nerve $\cdot$ Iatrogenic injury

B. M. Henry

bmhenry55@gmail.com

1 Department of Surgical and Biomedical Sciences, University of Perugia, Perugia, Italy

2 Department of Anatomy, Jagiellonian University Medical College, 12 Kopernika Street, 31-034 Kraków, Poland

3 Department of Surgical Sciences, The University of Rome "La Sapienza", Rome, Italy

4 Tift College of Education, Mercer University, Atlanta, GA, USA

\section{Introduction}

Knowledge of the course of nerves in the inguinal region is essential for the treatment of hernia. Proper nerve identification during open hernia surgery can influence the incidence of postoperative chronic pain [1].

The inguinal canal runs through the muscles of the abdominal wall in an oblique direction, downward and medially, allowing for the passage of the spermatic cord (male) and round ligament (female). It is bounded by the transverse fascia posteriorly, the aponeurosis of external oblique anteriorly, the inguinal ligament inferiorly, and the bottom edge of the internal oblique and transverse abdominal muscles superiorly [2]. The canal has two openings: the upper one (internal inguinal ring) and the lower one (external inguinal ring). 


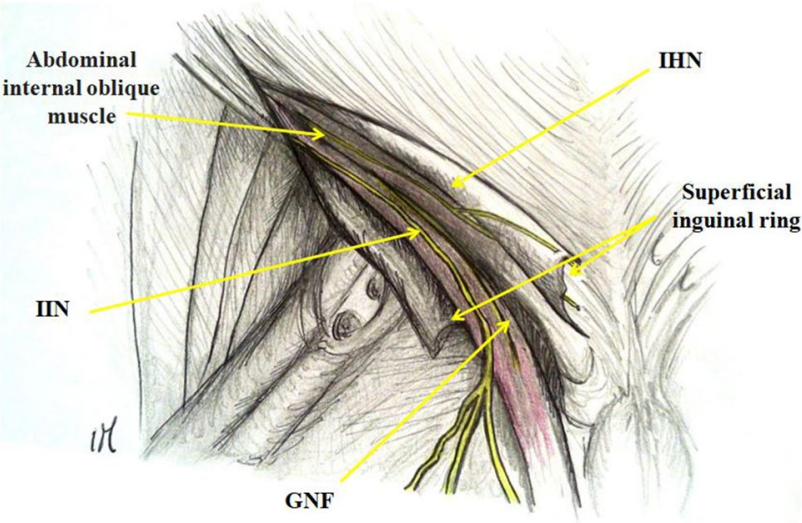

Fig. 1 Anatomy of the inguinal region

The inguinal canal is also crossed by the iliohypogastric (IHN), ilioinguinal (IIN) and the genital branch of the genitofemoral nerves (GNF) (Fig. 1). These nerves are the terminal branches of the lumbar plexus and innervate the abdominal muscle and the skin of genitals, buttock, and hypogastric region [3]. The IHN, IIN, and GNF are potentially at risk of iatrogenic injury during common surgical procedures, such as caesarean section, inguinal hernioplasty and most laparoscopic procedures.

Anatomical variants of the nerves in the inguinal region have been reported in the literature, but their prevalence is heterogeneous across different studies [4]. Patients who undergo inguinal hernioplasty may suffer from persistent postoperative pain, with an incidence that varies from 0.7 to $43.3 \%$ and with a rate of debilitating pain that varies from 0.5 to $6 \%[5,6]$. Previous research showed that failure to identify inguinal nerves is correlated with the presence of chronic pain [7]. Moreover, the incidence of this complication increases with the number of undetected nerves [2]. Having detailed knowledge on the inguinal nerves can significantly improve the safety and success rate of several surgical procedures besides inguinal hernia repair, such as varicocele surgery and ilioinguinal/iliohypogastric blocks with ultrasound-guided or landmark-based techniques [8-10].

The current European Hernia Society guidelines suggest the identification of the three inguinal nerves to decrease late postoperative pain, but in clinical practice, the fundamental question is: "Is it possible to identify every inguinal nerve during hernioplasty?" [10]. The aim of this systematic review and meta-analysis on inguinal nerves was to analyze and provide comprehensive data on their prevalence (identification rates), anatomical characteristics, and possible sources of heterogeneity, to decrease the risk of iatrogenic injury to these nerves during inguinal surgery.

\section{Materials and methods}

\section{Study selection}

A systematic review was performed on studies assessing the anatomical variations of inguinal nerves in accordance with the Preferred Reporting Items for Systematic Reviews and Meta-analyses (PRISMA) standards. The systematic literature search was conducted using the PubMed, Scopus and Web of Science database engines employing the terms: "inguinal" and "nerve" or "ilioinguinal" and "nerve" or "iliohypogastric" and "nerve" or "genitofemoral" and "nerve". No language or publication date restrictions were imposed.

Two authors (RC and MB) independently screened fulltext papers for eligibility. When multiple articles were published from a single study group and when overlapping study periods were reported, only the most recent article was considered to avoid duplication of data. The PubMed function "related articles" was used to broaden each search and the reference list of all potentially eligible studies was analyzed. To minimize retrieval bias, a manual search including the Science Citation Index Expanded, Scopus and Google Scholar databases was performed. The final decision on eligibility was reached by consensus between the two screening authors.

\section{Inclusion and exclusion criteria}

To be included in the present meta-analysis, a study had to report clear anatomical identification of inguinal nerves as primary or secondary outcomes in cadaveric or prospective operative studies. Case reports, editorials, conference abstracts, and studies reporting incomplete or irrelevant data were excluded.

A protocol for this meta-analysis was registered on PROSPERO: CRD42017074589 (http://www.crd.york. ac.uk/prospero).

\section{Data extraction}

We developed a data extraction sheet based on the Cochrane Consumers and Communication Review Group's data extraction template. Two authors (RC and MB) independently retrieved data from the included studies. A third author (JR) checked the extracted data. Disagreements were solved through discussion and, if necessary, by involving an independent fourth author (CR).

\section{Outcomes}

The primary outcome of interest was the prevalence (identification rate) of the inguinal nerves: IIN, IHN, or GNF. 
The following anatomical reference points were considered as secondary outcomes:

a. Distance of the emergence of the IIN from abdominal wall:

- inferiorly to the anterior superior iliac spine,

- medially to the anterior superior iliac spine.

b. Variations in the emergence of the IIN posteriorly to:

- the inguinal ligament,

- the anterior superior iliac spine (ASIS).

c. Aberrant origin of the IIN from the GNF.

d. IIN common trunk with the IHN.

e. Course of the IIN with regard to the spermatic cord:

- parallel,

- ventral.

f. Type of exit of IIN from inguinal canal:

- IIN exit through superficial inguinal ring (SIR)

- Acute infero-lateral angulation of the IIN in close contact with and parallel to the SIR fibers at exit.

- A plane superficial to the external oblique aponeurosis (EOA) having pierced it proximal to the SIR.

g. Mode of termination and branches.

\section{Statistical analysis}

Binomial pooled prevalence estimates (PPEs) (i.e., identification rates) for the IIN, IHN, GNF, and anatomical reference points were computed using MetaXL software (version 5.0). Other analyses were completed with SPSS 24.0. The $I^{2}$ statistic and its $95 \%$ confidence interval and Cochrane's $Q$ and significance level were reported as indicators of heterogeneity. We examined funnel and DOI plots for outcomes with ten or more studies. Where there was significant asymmetry in those plots, we conducted a sensitivity analysis between a random-effects model and an inverse variance fixed-effects model with a heterogeneity correction [11, 12] as suggested in Sterne et al. [13]. In addition, we conducted a leave-one-out sensitivity analysis for overall outcomes with 10 or more studies. In one study, we estimated the standard deviation from the range using the recommendations in Hozo, Djulbegovic, and Hozo [12]. Subgroups analyses were conducted for type of dissection (cadaveric or during hernioplasty), geographical region (Africa, Asia, Europe, North America, or South America), and the number of study centers (single center or multicenter). We also examined year and study sample size as possible sources of heterogeneity. An unweighted multiple regression analysis was carried out to identify the degree to which each of the following predictors, in concert, were associated with the IIN identification rate: type of dissection, geographical region, number of study centers, year of publication and study sample size.

\section{Results}

The PRISMA flow diagram for the systematic review is presented in Fig. 2. The initial search yielded 6878 potentially relevant articles. After removing 5014 duplicates and assessing titles/abstracts for eligibility, 1821 further articles were eventually excluded. Forty-eight studies were analyzed in full-text. Of these, 22 were excluded because the primary outcome of our review was not described. Finally, 26 articles were included in this systematic review and meta-analysis (Table 1) [2, 3, 7, 14-36].

Twenty-six included studies reported 5265 half-body examinations. Fourteen studies were performed during inguinal hernioplasty, 12 studies were performed during

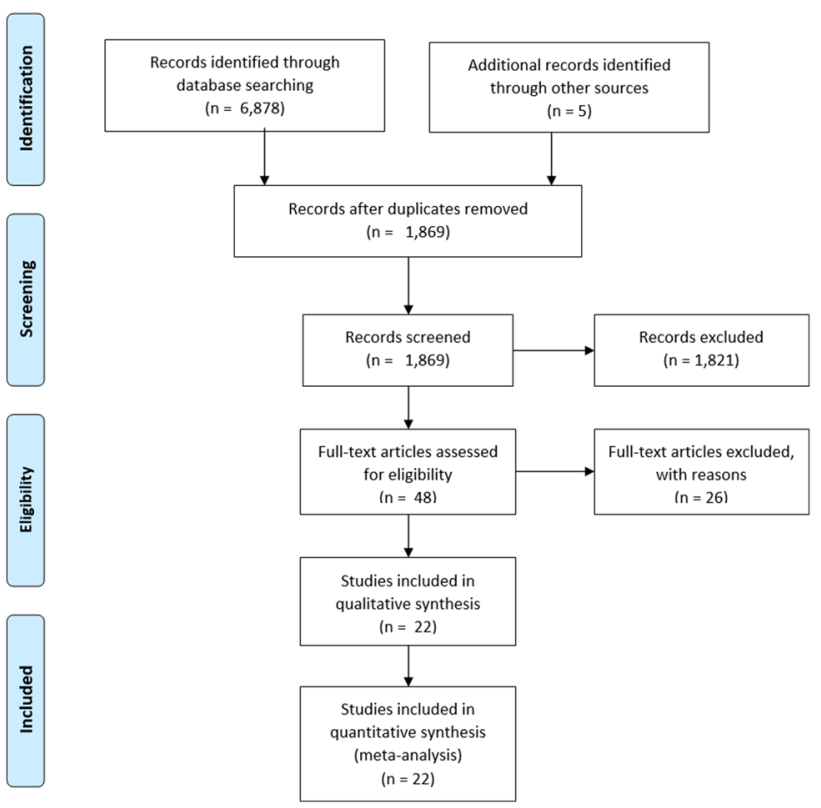

Fig. 2 PRISMA flow diagram 
Table 1 Included studies

\begin{tabular}{|c|c|c|c|c|}
\hline Author, year of publication & Country & Type of study & Single center/multicenter & $\begin{array}{l}n=(\# \\
\text { half-bodies } \\
\text { studied })\end{array}$ \\
\hline \multirow[t]{2}{*}{ Mendes 2016 [14] } & \multirow[t]{2}{*}{ Brazil } & Cadaveric & Single center & 10 \\
\hline & & During hernioplasty & Single center & 29 \\
\hline Smeds 2016 [15] & $\begin{array}{l}\text { UK/Sweden/The Neth- } \\
\text { erlands }\end{array}$ & During hernioplasty & Multicenter & 507 \\
\hline Grossi 2015 [16] & Brazil & During hernioplasty & Single center & 38 \\
\hline Sanders 2014 [17] & UK/Sweden & During hernioplasty & Multicenter & 553 \\
\hline Pandhare 2013 [3] & India & Cadaveric & Single center & 40 \\
\hline Emeksiz 2013 [18] & Turkey & During hernioplasty & Single center & 116 \\
\hline Yıldız 2012 [19] & Turkey & Cadaveric & Single center & 34 \\
\hline Bischoff 2012 [20] & Denmark & During hernioplasty & Single center & 244 \\
\hline Klaasen 2011 [21] & USA & Cadaveric & Single center & 200 \\
\hline Ergül 2011 [22] & Turkey & During hernioplasty & Single center & 25 \\
\hline Smeds 2010 [23] & Sweden & During hernioplasty & Single center & 525 \\
\hline Ndiaye 2010 [24] & France & Cadaveric & Single center & 100 \\
\hline Lange 2009 [25] & The Netherlands & During hernioplasty & Single center & 40 \\
\hline Wijsmuller 2007 [2] & The Netherlands & Cadaveric & Single center & 18 \\
\hline Bartlett 2007 [26] & UK & During hernioplasty & Single center & 172 \\
\hline Mui 2006 [27] & China & During hernioplasty & Single center & 100 \\
\hline Alfieri 2006 [7] & Italy & During hernioplasty & Multicenter & 973 \\
\hline Picchio 2004 [28] & Italy & During hernioplasty & Single center & 813 \\
\hline Ducic 2004 [29] & USA & Cadaveric & Single center & 20 \\
\hline Al-dabbagh 2002 [30] & UK & During hernioplasty & Single center & 110 \\
\hline Rab 2001 [31] & USA & Cadaveric & Multicenter & 64 \\
\hline Diop 2000 [32] & Senegal & Cadaveric & Single center & 40 \\
\hline Ravichandran 2000 [33] & UK & During hernioplasty & Single center & 40 \\
\hline Mandelkow 1988 [34] & Germany & Cadaveric & Single center & 88 \\
\hline Salama 1983 [35] & France & Cadaveric & Single center & 25 \\
\hline Papadopoulos 1981 [36] & Greece & Cadaveric & Single center & 341 \\
\hline Total & & & & 5265 \\
\hline
\end{tabular}

cadaveric dissections. A study by Mendes et al. [14] reported two different dissection types: Mendes2016a was for cadaveric dissection; Mendes 2016b was for during hernioplasty-which were counted as independent effect measures for the purposes of analysis. All studies were prospective in design.

Table 2 Overall geographic localization and type of inguinal dissection

\begin{tabular}{|c|c|c|c|c|c|c|}
\hline \multirow[t]{3}{*}{ Continents } & \multicolumn{6}{|c|}{ Type of inguinal dissection } \\
\hline & \multicolumn{2}{|l|}{ Cadaveric } & \multicolumn{2}{|c|}{ Hernioplasty } & \multicolumn{2}{|l|}{ Total } \\
\hline & $\begin{array}{l}\text { Number of } \\
\text { studies }\end{array}$ & $n=$ half-bodies ( $\%$ within region) & $\begin{array}{l}\text { Number of } \\
\text { studies }\end{array}$ & $n=$ half-bodies ( $\%$ within region) & $\begin{array}{l}\text { Number of } \\
\text { studies }\end{array}$ & $\begin{array}{l}n=\text { half- } \\
\text { bodies ( } \% \text { of } \\
\text { total) }\end{array}$ \\
\hline Europe & 5 & $572(12.58 \%)$ & 10 & $3977(87.42 \%)$ & 15 & $4549(86.4 \%)$ \\
\hline Asia & 2 & $74(23.5 \%)$ & 3 & $241(76,5 \%)$ & 5 & $315(6 \%)$ \\
\hline South America & 1 & $10(13 \%)$ & $2 *$ & $67(87 \%)$ & $3 *$ & $77(1.5 \%)$ \\
\hline North America & 3 & $284(100 \%)$ & 0 & 0 & 3 & $284(5.4 \%)$ \\
\hline Africa & 1 & $40(100 \%)$ & 0 & 0 & 1 & $40(0.7 \%)$ \\
\hline Total & 12 & $980(18.62 \%$ of total $)$ & 14 & $4285(81.38 \%$ of total $)$ & 26 & 5265 \\
\hline
\end{tabular}

*One article includes cadaveric specimen and hernia repair in the same study which were counted as separate effect sizes 
Most studies were conducted in Europe (Table 2). Fifteen studies were performed in Europe, five in Asia, three in North America, two in South America (both studies from Brazil), and one in Africa. No studies were performed in Australasia (Table 3). The nerve identification rates at the inguinal canal were evaluated (Table 3 ).

\section{Meta-analysis on the identification rate of the ilioinguinal nerve}

Figure 3 shows the identification rate of the IIN. A total of 21 studies and 3773 half-bodies were analyzed using a random-effects model (Table 4). The overall identification rate was $94.4 \%$ (95\% CI 89.5-97.9). In a leave-one-out sensitivity analysis, the identification rates varied slightly from 93.7 to $95.2 \%$. The funnel plot and DOI plot indicated major asymmetry; therefore, we also examined fixed-effect model with heterogeneity correction.

Under the fixed-effect model with a heterogeneity correction, which gives more weight to large studies like Alfieri et al. [7] $(n=525)$ and Smeds et al. [15] $(n=973)$, the identification rate was $87.0 \%$ (95\% CI 76.7\%-95.8\%) (Fig. 4). The median sample size for studies included in this analysis was 40. A follow-up unweighted multiple regression analysis showed that study sample size $(\beta=-0.74, p=.036)$ was the only statistically significant predictor for lower identification out of the following variables: sample size, year, region, number of centers, and type of dissection, (for the whole model: $\left.R^{2}=0.56, F(8,12)=1.94, p=.146\right)$. See Fig. 5 for a partial regression plot between sample size and PPE. The outlier in the bottom left of Fig. 5 was Lange et al. [25] — a study with a small sample size $(n=40)$ and a low identification rate $(75.0 \%, 95 \%$ CI $60.3 \%-87.4 \%)$. For the remainder of this analysis, we assumed that the sample size/ prevalence relationship was a source of bias and, therefore, we described results for both random-effect and fixed-effect models.

\section{Meta-analysis on the identification rate of the iliohypogastric nerve}

Figures 6 (random-effects model) and 7 (fixed-effects model) show the identification rate for IHN. A total of 15 studies and 4187 half-bodies were analyzed. The overall identification rate for the IHN was $86.7 \%$ (95\% CI 78.3\%-93.3\%) and $76.3 \%$ (95\% CI 62.5\%-88.9\%) using a random-effects model and fixed-effects model, respectively. In a leave-one-out sensitivity analysis, the identification rates varied slightly from 84.4 to $88.1 \%$ for a random-effects model and from 74.3 to $80.9 \%$ for a fixed-effects model. A visual analysis of a funnel plot and DOI plot indicated marked asymmetry; we also noted that there was a negative relationship between prevalence and sample size-similar to the IIN outcome. Table 5

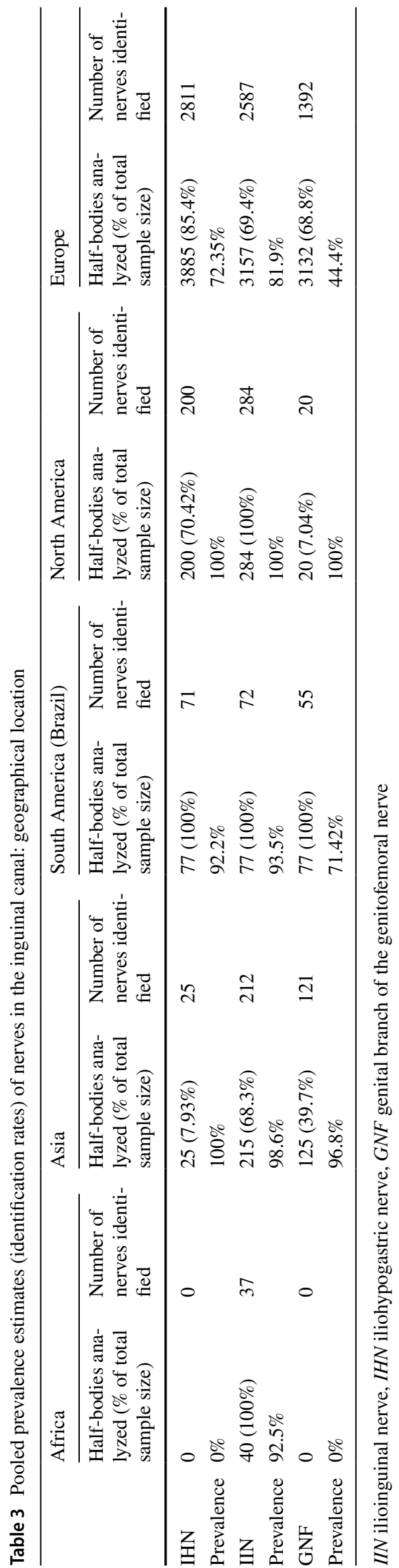


Fig. 3 Pooled prevalence estimates (identification rates) of the IIN under a random-effects model

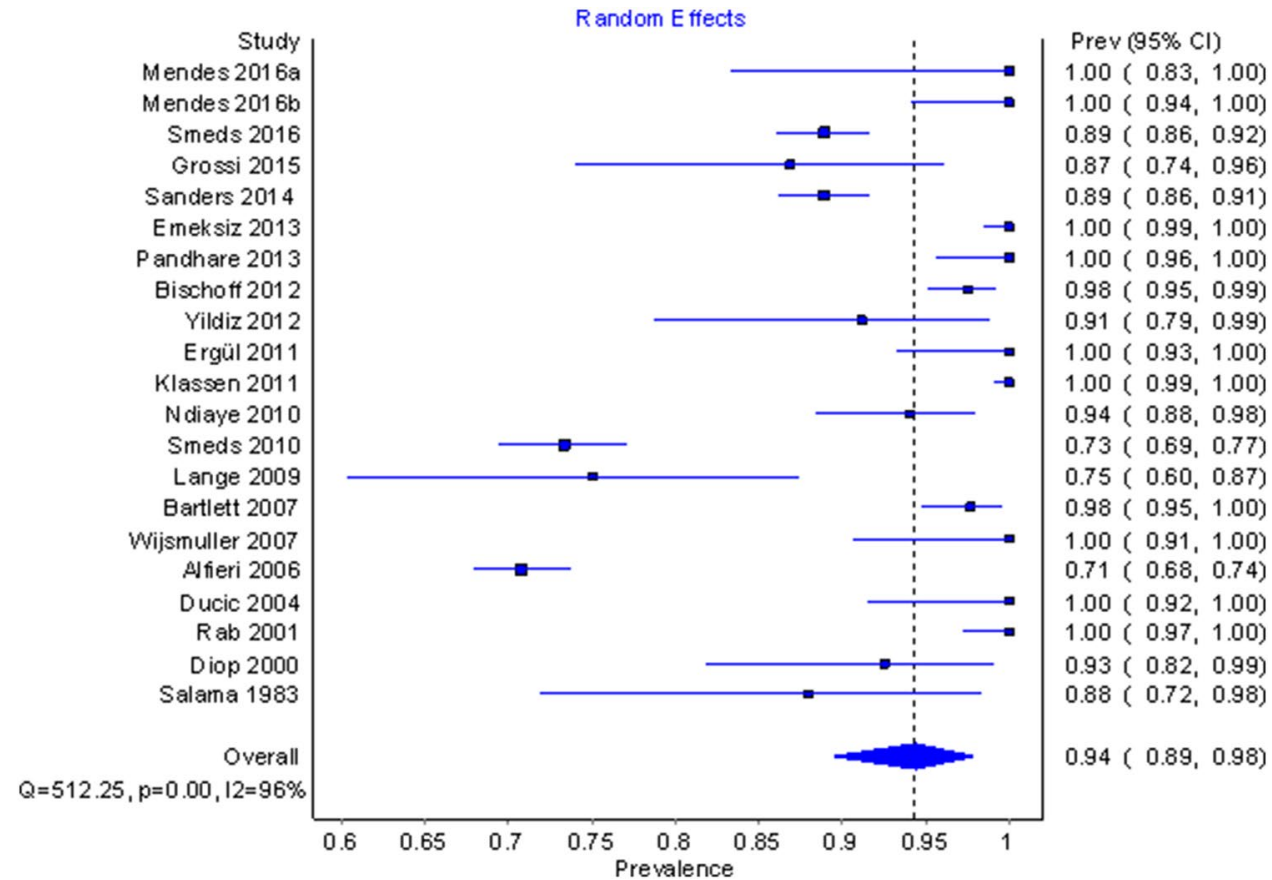

Table 4 Pooled prevalence estimates (identification rates) of the IIN

\begin{tabular}{|c|c|c|c|c|c|c|}
\hline Study Group & $N$ & Half-bodies & $\begin{array}{l}\text { PPE\% (95\% CI) } \\
\text { Random }\end{array}$ & $\begin{array}{l}\text { PPE\% }(95 \% \text { CI }) \\
\text { Fixed }\end{array}$ & $I^{2}(95 \% \mathrm{CI})$ & $Q$ \\
\hline All Studies & 21 & 3773 & $94.4(89.5-97.9)$ & $87.0(76.7-95.8)$ & $96.1(95.0-96.9)$ & $512.2^{* * * *}$ \\
\hline \multicolumn{7}{|l|}{ Type of dissection } \\
\hline Cadaveric & 10 & 551 & $97.3(93.6-99.5)$ & $98.1(94.6-1.00)$ & $70.6(43.8-84.6)$ & $30.6^{* * *}$ \\
\hline During hernioplasty & 11 & 3222 & $91.4(84.3-96.9)$ & $84.3(72.4-94.5)$ & $97.1(96.1,97.9)$ & $346.9^{* * * *}$ \\
\hline \multicolumn{7}{|l|}{ Study center } \\
\hline Single center & 17 & 1676 & 95.9 (89.9-99.2) & 92.3 (82.1-99.7) & $94.2(92.0-95.7)$ & $274.4^{* * * *}$ \\
\hline Multicenter & 4 & 2097 & $90.1(77.9-98.1)$ & $82.0(66.3-95.3)$ & $97.9(96.6-98.8)$ & $146.2^{* * *}$ \\
\hline \multicolumn{7}{|l|}{ Geographic region } \\
\hline Asia & 1 & 40 & $91.8^{\mathrm{a}}(81.9-99.0)$ & $91.8^{\mathrm{a}}(81.9-99.0)$ & $\mathrm{NC}$ & $\mathrm{NC}$ \\
\hline Africa & 1 & 40 & $99.4^{\mathrm{a}}(95.7-1.00)$ & $99.4^{\mathrm{a}}(95.7-1.00)$ & $\mathrm{NC}$ & $\mathrm{NC}$ \\
\hline Europe & 13 & 3332 & $91.5(84.9-96.5)$ & $84.6(73.5-94.2)$ & $96.6(95.4-97.5)$ & $351.5^{* * *}$ \\
\hline North America & 3 & 284 & $99.8^{\mathrm{b}}(99.1-100.0)$ & $99.8^{\mathrm{b}}(99.1-100.0)$ & $0.0(0.0-57.9)$ & 0.50 \\
\hline South America (Brazil) & 2 & 77 & $95.2(84.2-100.0)$ & $94.7(81.9-1.00)$ & $67.3(0.0-90.6)$ & $6.13^{*}$ \\
\hline
\end{tabular}

$P P E$ pooled prevalence estimate, $N C$ not computable because there was only one study in this group

$* p<.05, * * p<.01, * * * p<.001$

${ }^{a}$ Fixed- and random-effects estimates are identical because there was only one study in this subgroup

${ }^{b}$ Fixed- and random-effect estimates are identical because the study-level prevalence rates were all $100.0 \%$

shows the results of the overall and subgroup analyses for the IHN. The identification rates ranged from $64.5 \%(95 \%$ CI $58.5 \%-70.4 \%$ ) for multicenter studies to $99.9 \%$ (95\% CI 99.1\%-100.0\%) for single center studies. As in the IIN outcome, there was a large, statistically significant amount of heterogeneity overall and within subgroups.

\section{Meta-analysis on the identification rate of the genital branch of the genitofemoral nerve}

Figures 8 and 9 and Table 6 show the results for the GNF. Fifteen studies and 3354 half-bodies were included. The identification rates for random-effects and fixed-effects models were $69.1 \%$ (95\% CI $53.1 \%-83.0 \%$ ) and $47.8 \%$ (95\% CI $22.8 \%-73.0 \%$ ), respectively. A leave-one-out 

estimates (identification rates) of the IIN under a fixed-effects model with heterogeneity correction
Fig. 4 Pooled prevalence

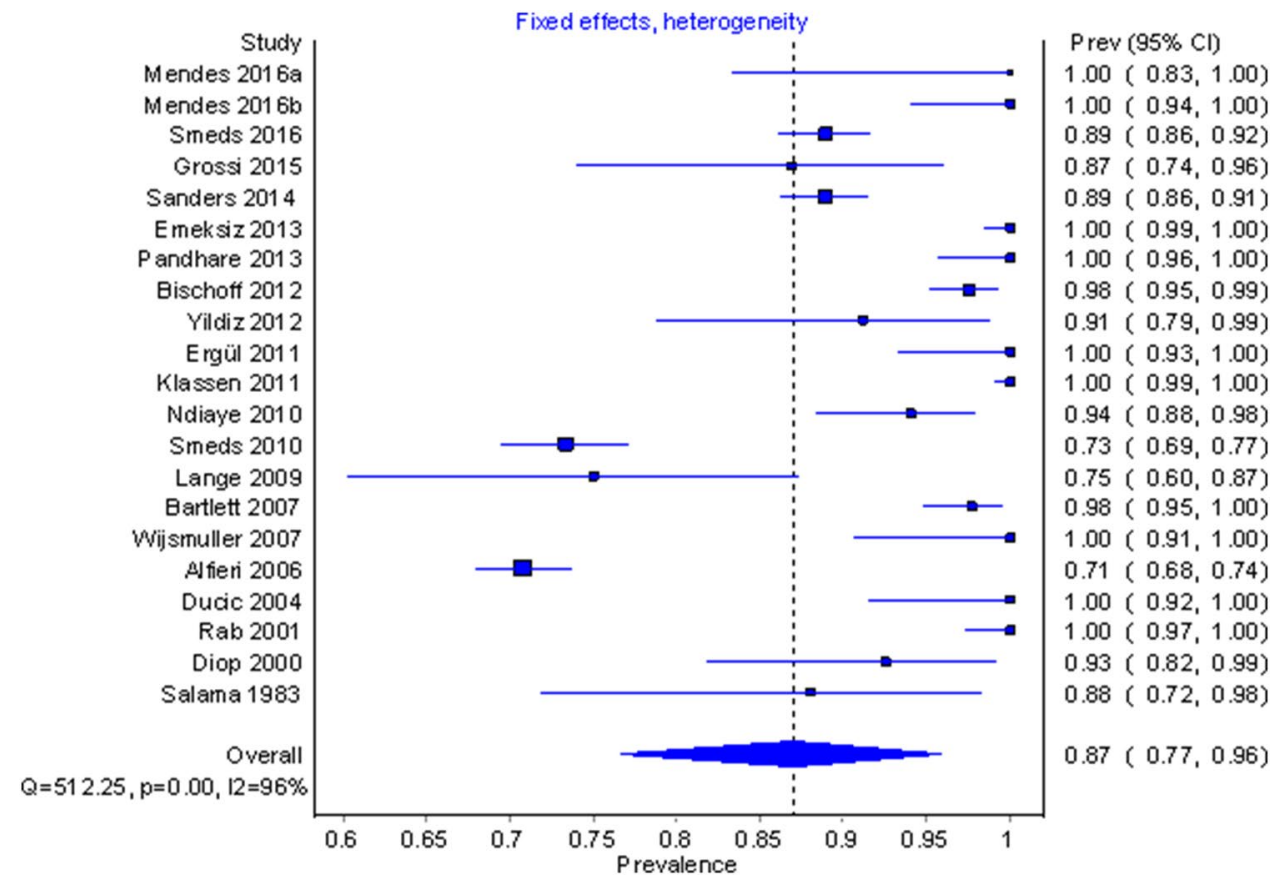

Meta-analysis of anatomical reference points

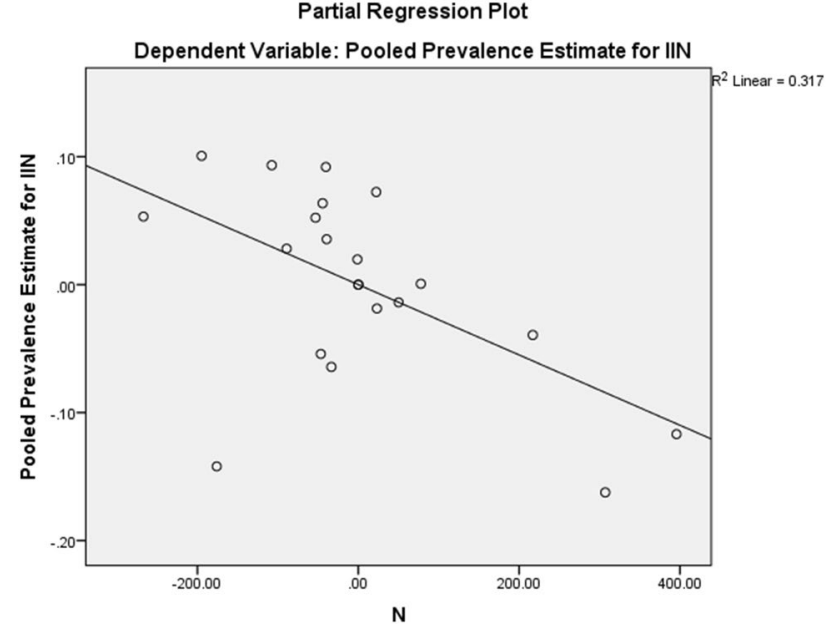

Fig. 5 Partial regression plot of sample size and pooled prevalence estimates of IIN when controlling for region, type of dissection, and number of centers. Note that values are mean-centered at zero. The outlier in the bottom left corner is Lange (2014) - a small sample size study $(N=40)$ with a low prevalence estimate $(75 \%)$

sensitivity results ranged from 64.8 to $73.6 \%$ for a randomeffects model and from 44.6 to $54.9 \%$ for a fixed-effects model. There was a large and statistically significant amount of heterogeneity overall and within subgroups. As with other outcomes, a visual analysis of funnel and DOI plots indicated irregularity and provided evidence that larger studies tended to have smaller prevalence.
Table 7 presents all secondary endpoints including the pooled estimates of distance of the point of the nerve emergence in relationship to the anatomic landmarks for the IIN, which was located inferior to the ASIS, medially to the ASIS, and the inguinal ligament. In one study [33], the range was reported instead of the standard deviation. Therefore, we estimated the standard deviation from the range using the guidelines in Hozo et al. [12] assuming an underlying normal distribution of nerve lengths. The distance from IIN emergence inferior to the ASIS was $2.8 \mathrm{~cm}(2.65-2.95)$ and medially to the ASIS was $3.62 \mathrm{~cm}$ (3.04-4.19). For nerve length outcomes, there was a large and statistically significant amount of heterogeneity. When computable, the heterogeneity estimates for other reference points were also large and statistically significant.

\section{Discussion}

Inguinal hernia repair is one of the most commonly performed surgical procedures. Nowadays, the most frequent hernia repair is in the outpatient setting, which requires the use of local anesthesia, and the most frequent postoperative complication is late pain in the inguinal region [10]. Failure to identify inguinal nerves during the surgery has been correlated with the higher incidence of postoperative pain [7].

In this review we included 26 studies with 5265 halfbodies examinations. Fourteen studies were performed 
Fig. 6 Pooled prevalence estimates (identification rates) of the IHN under a random-effects model

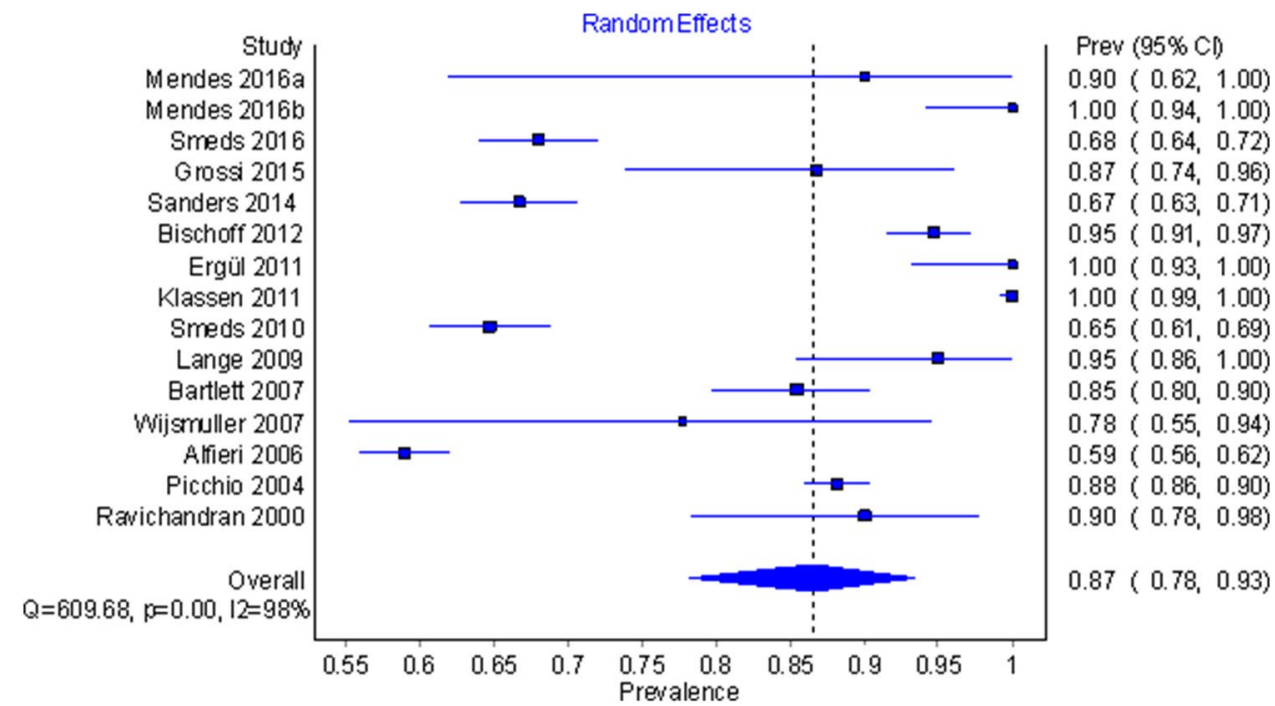

Fig. 7 Pooled prevalence estimates (identification rates) of the IHN under a fixed-effects model with heterogeneity correction

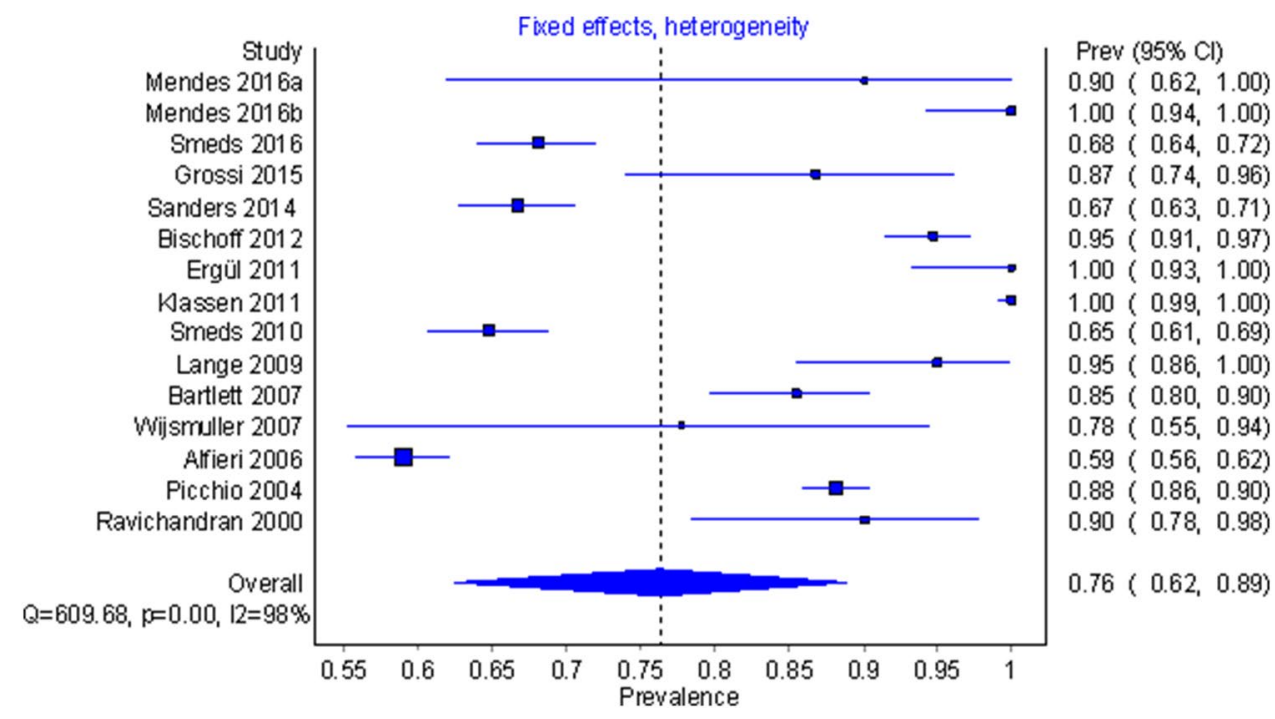

during inguinal hernioplasty and 12 during cadaveric dissections. We analyzed the identification rates of the nerves at the inguinal canal. The IIN nerve-identification rate was evaluated in 20 studies and its presence was reported in the $84.6 \%$ of the dissections. The IHN identification rate was evaluated in 14 studies and its presence was reported in $74.2 \%$ of the half-bodies' examinations. The identification rate of the GNF was evaluated in 14 studies and the presence of nerve was reported in $47.34 \%$ of the cases. The analysis of the identification rates show that it is not always possible to correctly locate all of inguinal nerves and that the nerve that is most difficult to locate is the GNF. Identification rates obtained in this study were lower than the rates reported by a narrative review performed on 13 studies: $96 \%$ for IIN, $94 \%$ for IHN and $90 \%$ for GNF [1]. In addition, the identification rate was higher in cadaveric studies (identification rate for IIN: $97.27 \%$, for
IHN: $97.8 \%$, for GNF: $37.83 \%$ ) than in inguinal hernioplasty studies (identification rate for IHN: $63.52 \%$, for IIN: $82.43 \%$, for GNF: $47.8 \%$ ) for all the nerves. This suggests in both cases the difficulty of identification of the GNF and that the different techniques used in anatomical and surgical procedures provide different outcomes.

Moreover, the identification rates of nerves varied across different geographic regions. There was a relatively higher identification rate of nerves reported in Asian studies and North American studies. In South America, the identification rate was very high for GNF. The data were very heterogeneous in other regions. In Africa, there was a identification rate of zero for IHN and GNF. In Europe, the identification rate was $4.4 \%$ for GNF, $72.35 \%$ for IHN, and $81.9 \%$ for IIN. We suspect the heterogeneity of patients and settings may have resulted in much of the heterogeneity between studies. 
Table 5 Pooled prevalence estimates (identification rates) of the IHN

\begin{tabular}{|c|c|c|c|c|c|c|}
\hline Study group & $N$ & Half-bodies & $\begin{array}{l}\text { PPE\% (95\% CI) } \\
\text { Random }\end{array}$ & $\begin{array}{l}\text { PPE\% }(95 \% \text { CI }) \\
\text { Fixed }\end{array}$ & $I^{2}(95 \% \mathrm{CI})$ & $Q$ \\
\hline All studies & 15 & 4187 & $86.7(78.3-93.3)$ & $76.3(62.5-88.9)$ & $97.7(97.1-98.2)$ & $609.68^{* * *}$ \\
\hline \multicolumn{7}{|l|}{ Type of dissection } \\
\hline Cadaveric & 3 & 228 & $91.8(66.8-1.00)$ & $99.2(74.5-1.00)$ & $89.3(70.9-96.1)$ & $18.68^{* * *}$ \\
\hline During hernioplasty & 12 & 3959 & $84.8(76.5-91.6)$ & $74.2(61.5-86.0)$ & $97.4(96.5-98.0)$ & $420.08^{* * *}$ \\
\hline \multicolumn{7}{|l|}{ Study center } \\
\hline Monocenter & 12 & 2154 & $91.4(83.2-97.2)$ & $86.6(72.2-98.1)$ & $96.0(94.5-97.2)$ & $227.66^{* * * *}$ \\
\hline Multicenter & 3 & 2033 & $64.5(58.5-70.4)$ & $63.4(57.1-69.5)$ & $87.1(63.1-95.5)$ & $15.45^{* * *}$ \\
\hline \multicolumn{7}{|l|}{ Geographic region } \\
\hline Asia $^{a}$ & - & - & - & - & - & - \\
\hline Africa $^{a}$ & - & - & - & - & - & - \\
\hline Europe & 11 & 3910 & $82.3(73.3-89.8)$ & $73.8(61.2-85.5)$ & $97.5(96.6-98.1)$ & $393.48^{* * * *}$ \\
\hline North America & 1 & 200 & $99.9^{\mathrm{b}}(99.1-100.0)$ & $99.9^{\mathrm{b}}(99.1-100.0)$ & $\mathrm{NC}$ & $\mathrm{NC}$ \\
\hline South America & 3 & 77 & $92.6(79.7-100.0)$ & $93.3(79.3-100.0)$ & $67.8(0.00-90.7)$ & $6.22^{*}$ \\
\hline
\end{tabular}

$P P E$ pooled prevalence estimate, $N C$ not computable because there was only one study in this group

$* p<.05, * * p<.01, * * * p<.001$

${ }^{\mathrm{a}}$ There were no studies of IHN prevalence from Asia or Africa

${ }^{\mathrm{b}}$ Fixed- and random-effects estimates are identical because the study-level prevalence rates were all $100.0 \%$ and there were was only one study

Fig. 8 Pooled prevalence estimates (identification rates) of the GNF under a random-effects model

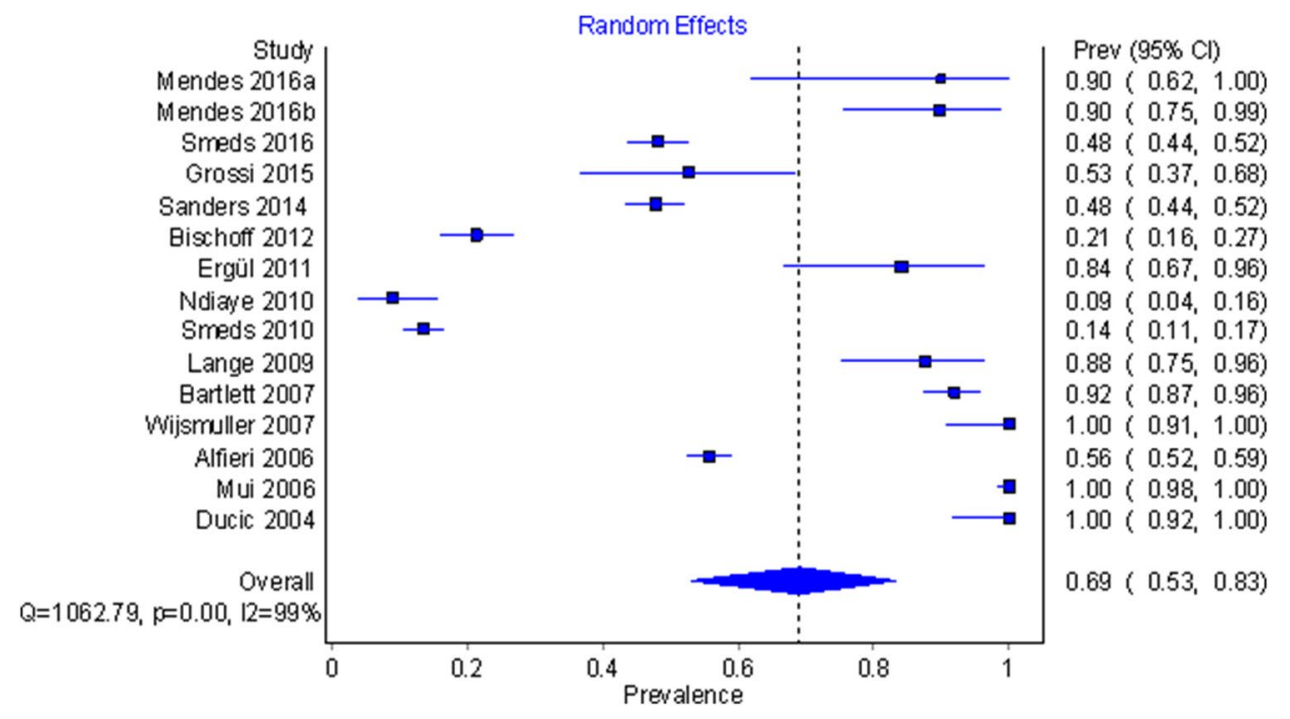

Finally, we also found that the study size was a predictor of the identification rate. Larger studies tended to have lower identification rates. Large sample size study being correlated with outcomes is a phenomenon that Sterne et al. [13] hypothesize could be the result of "interventions being implemented less thoroughly in larger studies, resulting in smaller effect estimates compared with smaller studies". The outlier study by Lange et al. [25] with a low nerve identification rate may be explained by the small sample size of patients included $(n=40)$. However, in this study, the authors used methods to increase reliability, with each identified nerve being photographed by the operating theatre nurse as proof which was rechecked by the surgeon and then reviewed by an anatomist.

In modern abdominal wall surgery, inguinal nerve identification plays an increasingly important role and represents a source of significant benefits. Nonetheless, inguinal nerve variants have always been a pitfall for surgeons and the fact that all structures cannot be located in all cases, as also this review demonstrated, has important repercussions for surgical practice. The European Hernia Society guidelines [10] recommend the identification of the three inguinal nerves (ilioinguinal, iliohypogastric and genital branch of the genitofemoral) for the reduction of late postoperative pain deriving from nerve injuries. 
Fig. 9 Pooled prevalence estimates (identification rates) of the GNF under a fixed-effects model with heterogeneity correction

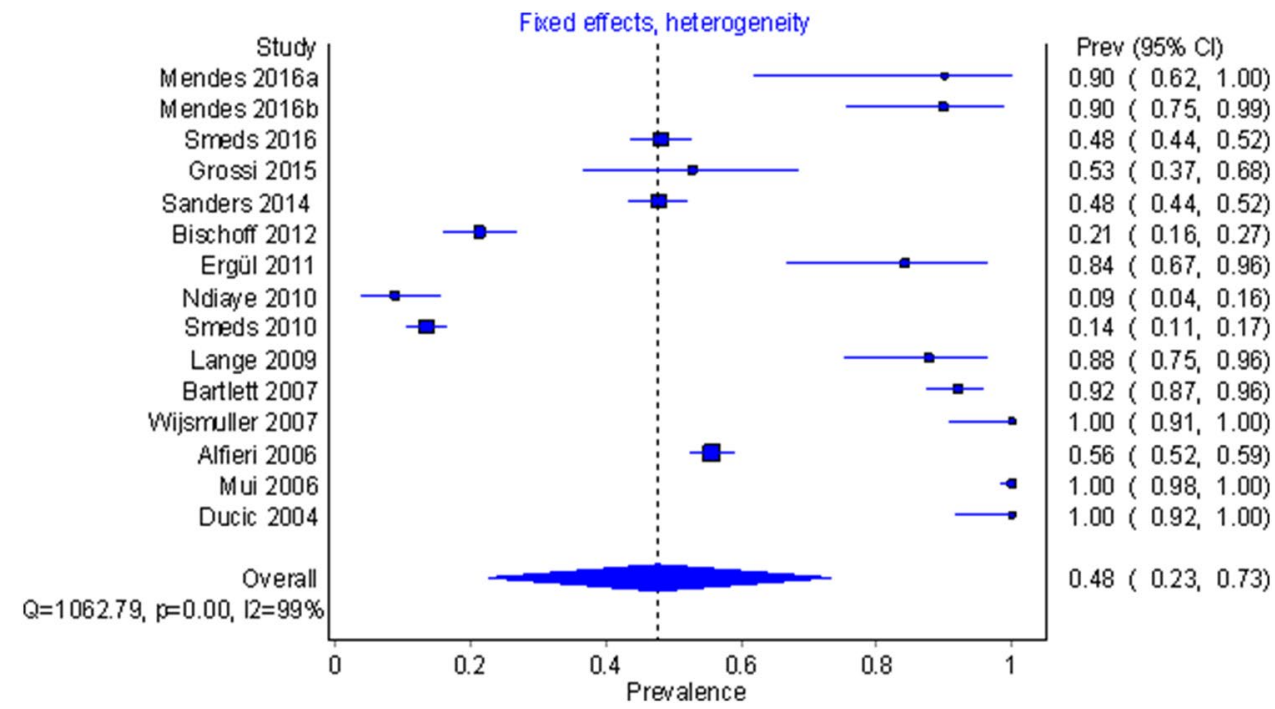

Table 6 Pooled prevalence estimates (identification rates) of the genital branch of the genitofemoral nerve

\begin{tabular}{|c|c|c|c|c|c|c|}
\hline Study group & $N$ & Half-bodies & $\begin{array}{l}\text { PPE\% (95\% CI) } \\
\text { Random }\end{array}$ & $\begin{array}{l}\text { PPE\% }(95 \% \text { CI) } \\
\text { Fixed }\end{array}$ & $I^{2}(95 \% \mathrm{CI})$ & $Q$ \\
\hline All studies & 15 & 3354 & $69.1(53.1-83.0)$ & $47.8(22.8-73.0)$ & 98.7 (98.4-98.9) & $1062.79^{* * * *}$ \\
\hline \multicolumn{7}{|l|}{ Type of dissection } \\
\hline Cadaveric & 4 & 148 & $79.6(0.0-100.0)$ & $38.7(0.0-100.0)$ & $98.1(96.9-98.9)$ & $160.20^{* * *}$ \\
\hline During hernioplasty & 11 & 3206 & $65.6(47.8-81.5)$ & $48.2(23.4-73.2)$ & $98.9(98.6-99.1)$ & $897.33^{* * * *}$ \\
\hline \multicolumn{7}{|l|}{ Study center } \\
\hline Single center & 12 & 1321 & $75.6(46.1-96.7)$ & $41.9(0.0-90.4)$ & $98.9(98.7-99.1)$ & $1020.63^{* * *}$ \\
\hline Multicenter & 2 & 2033 & $50.7(45.2-56.1)$ & $51.6(45.9-57.2)$ & $\mathrm{NC}$ & $11.92^{* *}$ \\
\hline \multicolumn{7}{|l|}{ Geographic region } \\
\hline Asia & 1 & 100 & $99.8^{\mathrm{a}}(98.3-1.00 .0)$ & $99.8^{\mathrm{a}}(98.3-1.00 .0)$ & $\mathrm{NC}$ & $\mathrm{NC}$ \\
\hline Africa $^{b}$ & - & - & - & - & - & - \\
\hline Europe & 10 & 3157 & $56.3(39.0-73.0)$ & $44.3(21.5-67.7)$ & $98.8(98.4-99.1)$ & $743.37^{* * * *}$ \\
\hline North America & 1 & 20 & $98.8^{\mathrm{a}}(91.5-100.0)$ & $98.8^{\mathrm{a}}(91.5-100.0)$ & $\mathrm{NC}$ & $\mathrm{NC}$ \\
\hline South America & 3 & 77 & $79.0(48.0-99.0)$ & $72.7(40.6-98.2)$ & $84.5(53.8-94.8)$ & $12.91^{* *}$ \\
\hline
\end{tabular}

$P P E$ pooled prevalence estimate, $N C$ not computable because there were two or fewer studies in this group ${ }^{*} p<.05, * * p<.01, * * * p<.001$

${ }^{a}$ Fixed- and random-effects estimates are identical because the study-level prevalences were all $100.0 \%$ and there were was only one study

${ }^{\mathrm{b}}$ There were no studies of IHN prevalence from Africa

The first description of abdominal pain after inguinal surgery was reported as "genitofemoral causalgia" from Magee in 1942 [37]. Heise and Starling [38] described the chronic pain after hernioplasty treated with partial or total prosthesis removal as "mesh inguinodynia". There are many controversies about the treatment of the identified inguinal nerves: Lichtenstein et al. [39] for example, proposed the preservation of the inguinal nerves after identification; other surgeons suggest the prophylactic neurectomy [40]. However, there is no evidence of the superiority of one of the two techniques in postoperative pain reduction.
Surgeons who mainly perform hernioplasty surgery have the best outcomes in terms of identifying nerve structures [41]. The success in surgical identification of the three nerves has been found to be largely associated with surgical skills [41], but sometimes some anatomical variations of the nerve topography makes the surgical identification difficult no matter the skill level, especially in the cases were some of inguinal nerves are not present. For these reasons, standardization of education and training in nerve identification in hernia surgery is needed [41, 42].

Our meta-analysis reported statistical analysis of nerve course variations to provide more reliable points of reference 
Table 7 Anatomical reference points for the ilioinguinal nerve

\begin{tabular}{|c|c|c|c|c|c|}
\hline Reference point & Studies & $N$ & PME (95\% CI) & $I^{2}(95 \% \mathrm{CI})$ & $Q$ \\
\hline \multicolumn{6}{|l|}{ Distance from the IIN emerged to } \\
\hline Inferior to the anterior superior iliac spine & 1 & 200 & $2.8 \mathrm{~cm}(2.65-2.95)$ & $\mathrm{NC}$ & $\mathrm{NC}$ \\
\hline Medially to the anterior superior iliac spine & 4 & 428 & $3.62 \mathrm{~cm}(3.04-4.19)$ & $92.8(84.9-96.6)$ & $41.83 * * *$ \\
\hline \multicolumn{6}{|l|}{ Variations in the emergence of the nerve } \\
\hline Posterior to the inguinal ligament & 2 & 140 & $19.6 \%(12.7-27.5)$ & $\mathrm{NC}$ & 1.18 \\
\hline Posterior to the anterior superior iliac spine & 2 & 140 & $4.5 \%(1.0-9.8)$ & $\mathrm{NC}$ & 1.37 \\
\hline Aberrant origin of the IIN from the genital branch of GNF & 2 & 130 & $2.5 \%(0.4-6.0)$ & $\mathrm{NC}$ & 0.95 \\
\hline Common trunk with the IHN & 5 & 365 & $10.0 \%(2.0-23.3)$ & $89.2(77.5-94.8)$ & $36.99 * * *$ \\
\hline \multicolumn{6}{|l|}{ Course of the IIN with regard to the spermatic cord } \\
\hline Parallel & 4 & 188 & $87.8 \%(46.6-100.0)$ & $96.9(94.5-98.3)$ & $97.31 * * *$ \\
\hline Ventrally & 4 & 188 & $57.2 \%(3.1-100.0)$ & $97.6(96.0-98.6)$ & $126.97 * * *$ \\
\hline \multicolumn{6}{|l|}{ Type of exit of IIN from inguinal canal } \\
\hline IIN exit through SIR & 5 & 276 & $64.5 \%(19.0-99.0)$ & $97.7(96.3-98.5)$ & $170.86^{* * *}$ \\
\hline $\begin{array}{l}\text { Acute infero-lateral angulation of the IIN in close contact with and } \\
\text { parallel to the SIR fibers at exit }\end{array}$ & 3 & 168 & $4.9 \%(0.0-20.5)$ & $89.7(72.2-96.2)$ & $19.36 * * *$ \\
\hline A plane superficial to the EOA having pierced it proximal to the SIR & 5 & 276 & $14.6 \%(7.0-24.1)$ & $70.5(24.8-88.4)$ & $13.55^{* * *}$ \\
\hline \multicolumn{6}{|l|}{ Mode of termination and branches } \\
\hline \multicolumn{6}{|l|}{ Unique trunks } \\
\hline Scrotal termination & 1 & 110 & $36.4 \%(27.6-45.6)$ & $\mathrm{NC}$ & $\mathrm{NC}$ \\
\hline Pubic termination & 1 & 110 & $3.6 \%(0.8-8.1)$ & $\mathrm{NC}$ & $\mathrm{NC}$ \\
\hline Femoral termination & 1 & 110 & $2.7 \%(0.3-6.8)$ & $\mathrm{NC}$ & $\mathrm{NC}$ \\
\hline Two branches & - & - & - & - & - \\
\hline Three branches & - & - & - & - & - \\
\hline Four branches & - & - & - & - & - \\
\hline
\end{tabular}

$P M E$ pooled mean estimate, $N C$ not computable because there were two or fewer studies in this group, SIR superficial inguinal ring, EOA external oblique aponeurosis, IIN ilioinguinal nerve, IHN iliohypogastric nerve, GNF genital branch of the genitofemoral nerve

A random-effects model was used for all outcomes

$* p<.05, * * p<.01, * * * p<.001$

for safe and correct local anesthesia, that would allow lowering of the incidence of chronic postoperative inguinal pain. For most nerve length outcomes, there was a large and statistically significant amount of heterogeneity. The data on anatomical reference points were in contrast with the data reported in classic anatomical textbooks, such as Clinical Anatomy by Regions [43]. Those authors suggest performing an anesthetic block of IIN and IHN $2.5 \mathrm{~cm}$ above the anterior superior iliac spine on the spinoumbilical line [43]. To ensure proper identification of inguinal nerves, ultrasonographic confirmation of their location should be attempted $[4,9]$. In cases of abnormal nerve courses, the successful application of blind anesthetic blocks may be impossible.

\section{Conclusion}

Our systematic review and meta-analysis provided the largest and most comprehensive up-to-date data on the identification rates of the inguinal nerves. The identification rates of the inguinal nerves in our study was lower than reported in literature. The lowest was found for the genital branch of genitofemoral nerve suggesting this nerve was the most difficult to identify. Moreover, the nerve topography results must be taken in account in the nerve sparing approach during hernioplasty. The knowledge about anatomy of inguinal nerves can facilitate their proper identification and reduce the risk of iatrogenic injury and postoperative pain.

Acknowledgements We would like to thank Alessandro Quintili, Bruno Perotti, Vito D'Andrea, and Ludovico Urbani who helped with this research project.

Author contributions RC, BMH, IM, and KAT were responsible for study design, interpretation of the results, and critical revisions of the manuscript for important intellectual content. PP, AS, and ML were responsible for study design, data collection, and manuscript drafting. JR was responsible for statistical analysis, interpretation of results, and manuscript drafting. All authors approved of the final written version.

Funding The authors received no specific funding for this research. 


\section{Compliance with ethical standards}

Conflict of interest R.C. declares that he has no conflict of interest. B.M.H. declares that he has no conflict of interest. I.M. declares that she has no conflict of interest. K.A.T. declares that he has no conflict of interest. P.P. declares that he has no conflict of interest. A.S. declares that she has no conflict of interest. M.L. declares that he has no conflict of interest. J.R. declares that he has no conflict of interest.

Ethical approval Approval from an institutional review board was not required for this study.

Human and animal rights This article does not contain any original data directly involving human participants, as it is a review of data obtained in previous studies.

Informed consent For this type of study, informed consent is not applicable.

Open Access This article is distributed under the terms of the Creative Commons Attribution 4.0 International License (http://creativeco mmons.org/licenses/by/4.0/), which permits unrestricted use, distribution, and reproduction in any medium, provided you give appropriate credit to the original author(s) and the source, provide a link to the Creative Commons license, and indicate if changes were made.

\section{References}

1. Alfieri S, Amid PK, Campanelli G et al (2011) International guidelines for prevention and management of post-operative chronic pain following inguinal hernia surgery. Hernia 15:239 249. https://doi.org/10.1007/s10029-011-0798-9

2. Wijsmuller AR, Lange JFM, Kleinrensink GJ et al (2007) Nerve-identifying inguinal hernia repair: a surgical anatomical study. World J Surg 31:414-420. https://doi.org/10.1007/s0026 8-006-0376-y

3. Pandhare SR, Gaikwad AP (2013) Anatomical study of ilioinguinal nerve and its clinical correlation. Int J Curr Res Rev 5:69-75

4. Frassanito L, Zanfini BA, Pitoni S et al (2018) Ultrasound-guided genitofemoral nerve block for inguinal hernia repair in the male adult: a randomized controlled pilot study. Minerva Anestesiol 84:189-195. https://doi.org/10.23736/S0375-9393.17.11948-6

5. Hsu W, Chen C-S, Lee H-C et al (2012) Preservation versus division of ilioinguinal nerve on open mesh repair of inguinal hernia: a meta-analysis of randomized controlled trials. World J Surg 36:2311-2319. https://doi.org/10.1007/s00268-012-1657-2

6. Barazanchi AWH, Fagan PVB, Smith BB, Hill AG (2016) Routine neurectomy of inguinal nerves during open onlay mesh hernia repair. Ann Surg 264:64-72. https://doi.org/10.1097/SLA.00000 00000001613

7. Alfieri S, Rotondi F, Di Giorgio A et al (2006) Influence of preservation versus division of ilioinguinal, iliohypogastric, and genital nerves during open mesh herniorrhaphy: prospective multicentric study of chronic pain. Ann Surg 243:553-558. https://doi. org/10.1097/01.sla.0000208435.40970.00

8. Yazigi A, Jabbour K, Jebara SM et al (2002) Bilateral ilioinguinal nerve block for ambulatory varicocele surgery. Ann Fr Anesth Reanim 21:710-712

9. Wang Y, Wu T, Terry MJ et al (2016) Improved perioperative analgesia with ultrasound-guided ilioinguinal/iliohypogastric nerve or transversus abdominis plane block for open inguinal surgery: a systematic review and meta-analysis of randomized controlled trials. J Phys Ther Sci 28:1055-1060. https://doi. org/10.1589/jpts.28.1055

10. Simons MP, Aufenacker T, Bay-Nielsen M et al (2009) European Hernia Society guidelines on the treatment of inguinal hernia in adult patients. Hernia 13:343-403. https://doi. org/10.1007/s10029-009-0529-7

11. Doi SAR, Barendregt JJ, Khan S et al (2015) Advances in the meta-analysis of heterogeneous clinical trials I: the inverse variance heterogeneity model. Contemp Clin Trial 45:130-138. https://doi.org/10.1016/j.cct.2015.05.009

12. Hozo SP, Djulbegovic B, Hozo I (2005) Estimating the mean and variance from the median, range, and the size of a sample. BMC Med Res Methodol 5:13. https://doi. org/10.1186/1471-2288-5-13

13. Sterne JAC, Sutton AJ, Ioannidis JPA et al (2011) Recommendations for examining and interpreting funnel plot asymmetry in meta-analyses of randomised controlled trials. BMJ 343:d4002. https://doi.org/10.1136/BMJ.D4002

14. Mendes CJL, Silva RA, Neto DPA et al (2016) Prospective study of the neurotopographic adequacy of transverse incision in Lichtenstein inguinal hernioplasty. Med (Baltimore) 95:e5335. https://doi.org/10.1097/MD.0000000000005335

15. Smeds S, Nienhuijs S, Kullman E et al (2016) Identification and management of the ilio-inguinal and ilio-hypogastric nerves in open inguinal hernia repair: benefits of self-gripping mesh. Hernia 20:33-41. https://doi.org/10.1007/s 10029-015-1372-7

16. Grossi JVM, Cavazzola LT, Breigeiron R (2015) Inguinal hernia repair: can one identify the three main nerves of the region? Rev Col Bras Cir 42:149-153. https://doi.org/10.1590/0100-69912 015003004

17. Sanders DL, Nienhuijs S, Ziprin P et al (2014) Randomized clinical trial comparing self-gripping mesh with suture fixation of lightweight polypropylene mesh in open inguinal hernia repair. Br J Surg 101:1373-1382. https://doi.org/10.1002/bjs.9598

18. Emeksiz S, Ozden H, Guven G (2013) Effects of variable courses of inguinal nerves on pain in patients undergoing lichtenstein repair for inguinal hernia: preliminary results. Acta Chir Belg 113:196-202. https://doi.org/10.1080/00015458.2013.11680911

19. Yildiz S, Kocabiyik N, Coskun K et al (2012) Association of anatomical variations of ilioinguinal nerve with inguinal hernia repair. Gulhane Med J 54:155. https://doi.org/10.5455/gulha ne. 18911

20. Bischoff JM, Aasvang EK, Kehlet H, Werner MU (2012) Does nerve identification during open inguinal herniorrhaphy reduce the risk of nerve damage and persistent pain? Hernia 16:573-577. https://doi.org/10.1007/s10029-012-0946-x

21. Klaassen Z, Marshall E, Tubbs RS et al (2011) Anatomy of the ilioinguinal and iliohypogastric nerves with observations of their spinal nerve contributions. Clin Anat 24:454-461. https://doi. org/10.1002/ca.21098

22. Ergül Z, Kulaçoğlu H, Sen T et al (2011) A short postgraduate anatomy course may improve the junior surgical residents' anatomy knowledge for the nerves of the inguinal region. Chirurgia (Bucur) 106:599-603

23. Smeds S, Löfström L, Eriksson O (2010) Influence of nerve identification and the resection of nerves "at risk" on postoperative pain in open inguinal hernia repair. Hernia 14:265-270. https:// doi.org/10.1007/s10029-010-0632-9

24. Ndiaye A, Diop M, Ndoye JM et al (2010) Emergence and distribution of the ilioinguinal nerve in the inguinal region: applications to the ilioinguinal anaesthetic block (about 100 dissections). Surg Radiol Anat 32:55-62. https://doi.org/10.1007/s0027 6-009-0549-0

25. Lange JFM, Wijsmuller AR, van Geldere D et al (2009) Feasibility study of three-nerve-recognizing Lichtenstein procedure for 
inguinal hernia. Br J Surg 96:1210-1214. https://doi.org/10.1002/ bjs.6698

26. Bartlett DC, Porter C, Kingsnorth AN (2007) A pragmatic approach to cutaneous nerve division during open inguinal hernia repair. Hernia 11:243-246. https://doi.org/10.1007/s 1002 9-007-0209-4

27. Lik-Man Mui W, Ng CSH, Ming-Kit Fung T et al (2006) Prophylactic ilioinguinal neurectomy in open inguinal hernia repair. Ann Surg 244:27-33. https://doi.org/10.1097/01.sla.0000217691 $.81562 .7 \mathrm{e}$

28. Picchio M, Palimento D, Attanasio U et al (2004) Randomized controlled trial of preservation or elective division of ilioinguinal nerve on open inguinal hernia repair with polypropylene mesh. Arch Surg 139:755. https://doi.org/10.1001/archsurg.139.7.755

29. Ducic I, Dellon AL (2004) Testicular pain after inguinal hernia repair: an approach to resection of the genital branch of genitofemoral nerve. J Am Coll Surg 198:181-184. https://doi. org/10.1016/j.jamcollsurg.2003.09.025

30. Al-dabbagh AKR (2002) Anatomical variations of the inguinal nerves and risks of injury in 110 hernia repairs. Surg Radiol Anat 24:102-107

31. Rab M, Ebmer And J, Dellon AL (2001) Anatomic variability of the ilioinguinal and genitofemoral nerve: implications for the treatment of groin pain. Plast Reconstr Surg 108:1618-1623

32. Diop M, Dia A, Ndiaye A et al (2000) Emergence and course of the ilioinguinal nerve of the groin. Morphologie 84:29-32

33. Ravichandran D, Kalambe BG, Pain JA (2000) Pilot randomized controlled study of preservation or division of ilioinguinal nerve in open mesh repair of inguinal hernia. Br J Surg 87:1166-1167. https://doi.org/10.1046/j.1365-2168.2000.01529.x

34. Mandelkow H, Loeweneck H (1988) The iliohypogastric and ilioinguinal nerves. Distribution in the abdominal wall, danger areas in surgical incisions in the inguinal and pubic regions and reflected visceral pain in their dermatomes. Surg Radiol Anat 10:145-149

35. Salama J, Sarfati E, Chevrel JP (1983) The anatomical bases of nerve lesions arising during the reduction of inguinal hernia. Anat Clin 5:75-81. https://doi.org/10.1007/BF01798977

36. Papadopoulos NJ, Katritsis ED (1981) Some observations on the course and relations of the iliohypogastric and ilioinguinal nerves (based on 348 specimens). Anat Anz 149:357-364

37. Magee RK (1942) Genitofemoral causalgia: (a new syndrome). Can Med Assoc J 46:326-329

38. Heise CP, Starling JR (1998) Mesh inguinodynia: a new clinical syndrome after inguinal herniorrhaphy? J Am Coll Surg 187:514-518

39. Lichtenstein IL, Shulman AG, Amid PK, Montllor MM (1989) The tension-free hernioplasty. Am J Surg 157:188-193

40. Chen DC, Amid PK (2012) Questioning the favorable influence of nerve identification during open inguinal herniorrhaphy is misleading and may increases the risk of surgeons' liability. Hernia 16:579-581. https://doi.org/10.1007/s10029-012-0967-5

41. Robson AJ, Wallace CG, Sharma AK et al (2004) Effects of training and supervision on recurrence rate after inguinal hernia repair. Br J Surg 91:774-777. https://doi.org/10.1002/bjs.4540

42. Lorenz R, Stechemesser B, Reinpold W et al (2017) Development of a standardized curriculum concept for continuing training in hernia surgery: German Hernia School. Hernia 21:153-162. https ://doi.org/10.1007/s10029-016-1566-7

43. Snell RS (2012) The abdomen: the abdominal wall. In: Snell RS (ed) Clinical anatomy by regions, 9th edn. Lippincott William \& Willkins, Philadelphia, pp 113-155 